\title{
TGF-ß/activin/nodal signal regulates the fate of human embryonic stem cells
}

\author{
Zhao $\mathrm{Wu}^{1}$, Wei Zhang ${ }^{1}$, Lei Xiao ${ }^{1}$ \\ ${ }^{1}$ Key Laboratory of Stem Cell Biology, Institute of Biochemistry and Cell Biology, the Cell Bank/Stem Cell bank, Shanghai \\ Institutes for Biological Sciences, Chinese Academy of Sciences, Shanghai 200031, China
}

The commitment of cells to the inner cell mass or trophectoderm in preimplantation blastocysts is the first segregation of cell lineages in the developing human embryo. The exogenous signals that control the fate determination between inner cell mass and trophectoderm remain obscure. Human embryonic stem cells (hESCs) provide a new model for the study of human developmental biology. We previously showed that TGF-ß/Activin/ Nodal signaling maintains the pluripotency of hESCs, which are derivatives of the inner cell mass of human blastocyst. Here we further demonstrate that TGF- $\beta$ /activin/nodal signaling also regulate the fate of hESCs. Our data also suggests the TGF- $\beta$ /activin/nodal signaling be the key switcher in the fate determination of cells at early stage of human embryonic development.

Cell Research (2008) 18:s30. doi: 10.1038/cr.2008.120; published online 4 August 2008

Correspondence: Lei Xiao

E-mail: leixiao@sibs.ac.cn

Lei Xiao, $\mathrm{PhD}$, received a $\mathrm{PhD}$ in biology from Munich University in 2003. Dr Xiao completed his postdoctoral training in Johns Hopkins University, School of Medicine in 2005. He is a Principal Investigator of
Shanghai Institute of Biochemistry and Cell Biology and the director of the Cell Bank/Stem Cell Bank, Shanghai Institute for Biological Sciences, Chinese Academy of Sciences. Dr Xiao's research focuses on human embryonic stem (ES) cells, which have the potential to differentiate into all other cell types and might be used for regeneration medicine. 